\title{
Stabilization of a thoracolumbar flexion-distraction injury utilizing a temporary interspinous process construct: technical note
}

\author{
Scott C. Wagner, MD, and Christopher K. Kepler, MD, MBA \\ Department of Orthopaedic Surgery, Rothman Institute at Thomas Jefferson University, Philadelphia, Pennsylvania
}

\begin{abstract}
Many techniques for fixation in the thoracolumbar spine have been described. Occasionally, particularly during operative management of unstable ligamentous or bony injuries, temporizing fixation may be required. The authors report the case of a patient with a ligamentous thoracolumbar flexion-distraction injury who underwent reduction, posterior instrumentation and fusion, and temporary fixation of the destabilized segment utilizing a novel interspinous process screw/rod construct. This construct was stable after placement and allowed for traditional instrumentation to be placed without causing secondary injury to the spinal cord. To the authors' knowledge, this technique has not previously been described.
\end{abstract}

https://thejns.org/doi/abs/10.3171/2017.6.SPINE17487

KEY WORDS Chance fracture; temporary stabilization; spinous process; pedicle screws; lumbar; thoracic; surgical technique

$\mathrm{M}$ ULTIPLE methods for spinal stabilization have been described, including newer techniques such as those involving translaminar screws, ${ }^{1}$ intralaminar screws, ${ }^{5}$ and cortical screws. ${ }^{8}$ When permanent fixation cannot be immediately obtained, however, often it is necessary to place temporary stabilization to prevent catastrophic abnormal motion at a destabilized spinal segment. Here we report a case of an unstable, ligamentous thoracic flexion-distraction injury that was stabilized temporarily utilizing a spinous process-based spanning pedicle screw-and-rod construct; this method of temporary fixation allowed for placement of traditional pedicle screws, and completion of the final stabilization and decompression procedure.

\section{Case Report}

A 44-year-old man was involved in a motorcycle accident and sustained a T12-L1 ligamentous flexion-distraction injury, as well as bilateral lung contusions and a displaced fracture of the left midshaft ulna. On presentation, he exhibited mild weakness (Grade 4/5) in all motor groups distal to the level of his injury. Preoperative sagittal CT and
MRI scans are shown in Fig. 1. According to the AOSpine Thoracolumbar Injury Classification, this ligamentous disruption with displacement would be classified as a type C injury with an N3 modifier for incomplete spinal cord injury; this type of injury morphology is automatically assigned 8 points based on the AOSpine Thoracolumbar Injury Severity Score with an additional 4 points added for the presence of neurological dysfunction. ${ }^{3}$ A score of greater than 5 indicates an unstable injury that requires surgical stabilization; ${ }^{9}$ therefore, the patient gave consent for injury reduction and posterior thoracolumbar instrumented fusion and was taken urgently to the operating suite. Intraoperative neuromonitoring was used for the entirety of the procedure, including monitoring of prepositioning motor and sensory evoked potentials, which remained stable throughout the procedure. Surgical exposure commenced in a standard posterior manner, with subperiosteal dissection proceeding without difficulty. An intraoperative localizer image was obtained to ascertain the exposed vertebral levels; it was noted that in the prone position, the patient's flexion-distraction injury became more significantly displaced (Fig. 2). No changes were noted on neuromonitoring after positioning or during 

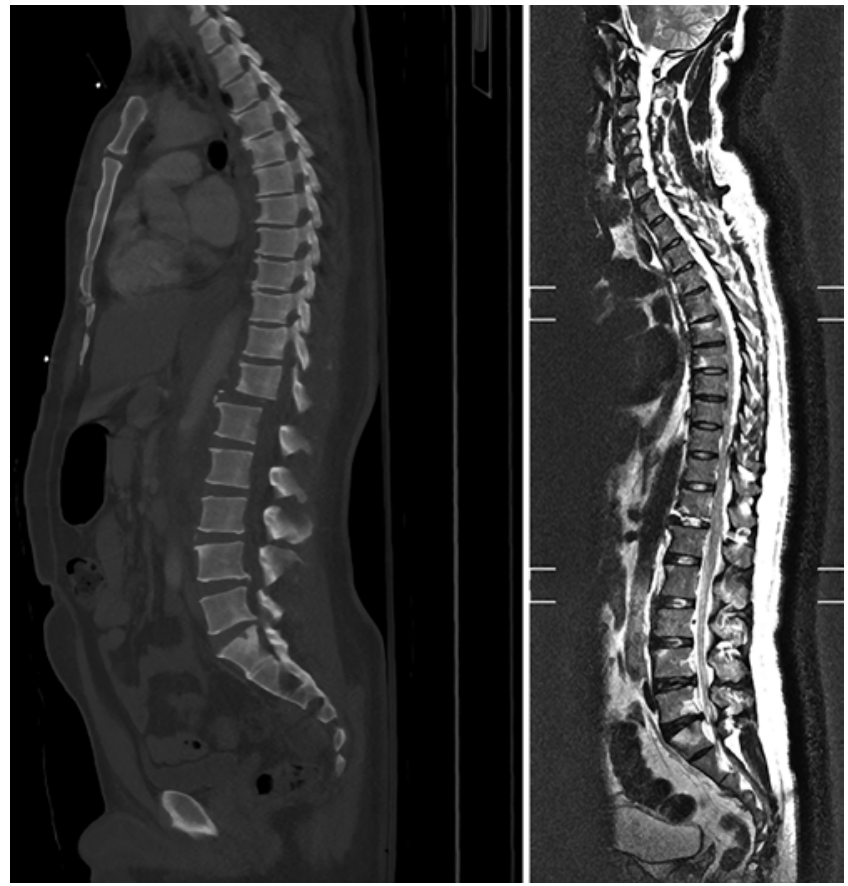

FIG. 1. Preoperative sagittal CT scan (left) and MR image (right) obtained upon presentation to the trauma bay. Both scans demonstrate an unstable ligamentous flexion-distraction injury at T12-L1 with evidence of displacement.

exposure. Exposure continued expeditiously, and once the fracture was exposed, the spinous processes of T-12 and L-1 were gripped with Kocher clamps, and the dislocated facet joints were reduced. However, after placement of a pedicle screw cephalad to the injury in T-12, reduction of the injury was difficult to maintain while attempting to drill for another screw caudal to the injury. Therefore, to minimize motion across the injured segment while placing the remaining screws, two holes were drilled and two 4.35 $\times 30-\mathrm{mm}$ polyaxial pedicle screws (DePuy Synthes Spine) were tapped into the spinous processes of T-11 and L-1 and were connected with a midline titanium rod. The initial drilling was performed with a $1.7-\mathrm{mm}$ burr tip, directed parallel and in-line with the spinous process. This tract was then cannulated utilizing a 4.35-mm tap for line-to-line fit. Screws were then placed with the lateral borders of the spinous processes under direct visualization to reduce the risk of breaching. The $4.35 \times 30-\mathrm{mm}$ size was chosen because it was the smallest screw in the set, and the screws were left prominent so as to prevent encroachment on the dorsal aspect of the spinal canal. A 5.5-mm pre-contoured rod was inserted into the polyaxial screw heads, and set screws were tightened in place with the torque-limited screwdriver and counter-torque device. An intraoperative radiograph and a photograph are shown in Figs. 3 and 4, respectively.

With this temporizing construct in place, reduction of the flexion-distraction injury was maintained, and the remaining pedicle screws were placed without difficulty. Upon completion of the final instrumentation, the interspinous construct was removed and a laminectomy performed at the level of the injury. Final plain radiographs are shown in Fig. 5. Postoperatively, the patient maintained

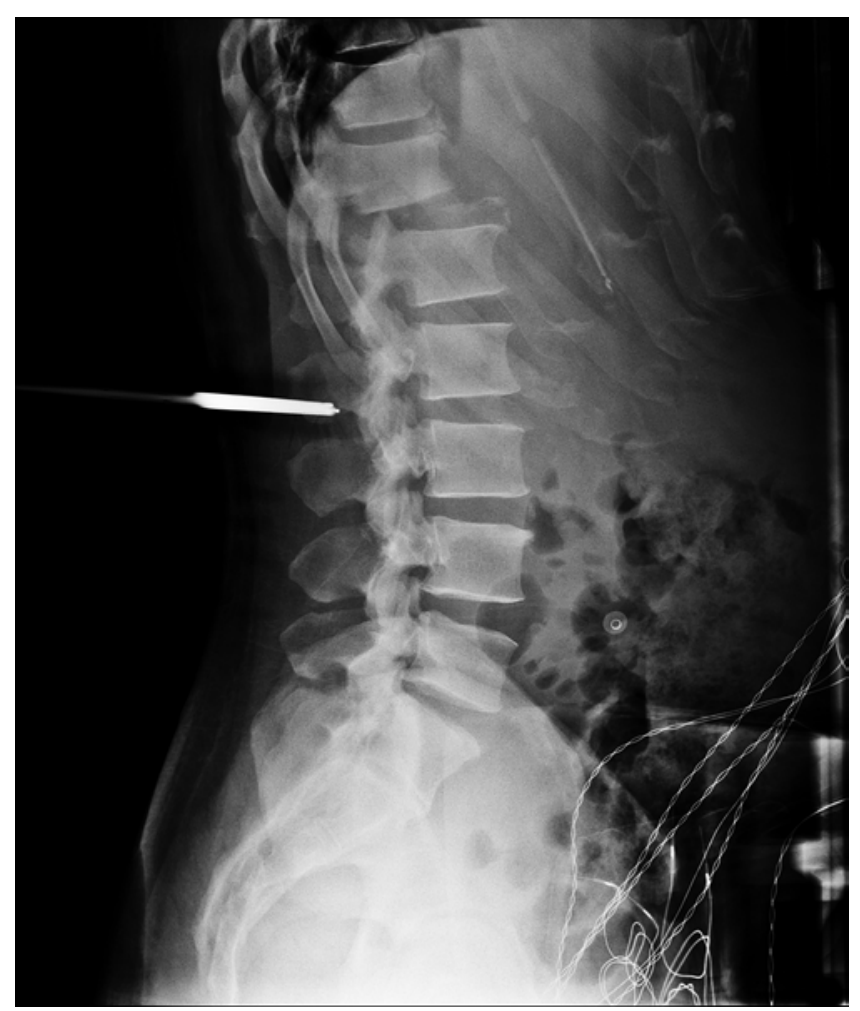

FIG. 2. Lateral intraoperative localization radiograph, obtained during exposure, demonstrating a Kocher clamp on the inferior aspect of $L-2$. Increased displacement of the T12-L1 flexion-distraction injury is apparent.

his level of neurological function, without any appreciable loss in motor or sensory function.

\section{Discussion}

To our knowledge, there are no previous reports describing the use of spanning spinous process instrumentation for temporary stabilization of a thoracolumbar injury. Despite the instability of the injury, the patient in our case had relatively well-preserved neurological function; typically, complete ligamentous disruptions are associated with a high rate of neurological compromise. ${ }^{6}$ It is also unclear how the change in positioning and alignment did not yield any changes in intraoperative neuromonitoring of motor and sensory evoked potentials, but because prepositioning baseline neuromonitoring had been obtained and no changes were observed postpositioning, no consideration was given to performing a Stagnara wake-up test or returning the patient to the supine position. This case also highlights the importance of intraoperative neuromonitoring; rather than advocating positioning prone patients in a brace or by another rigid mechanism, with maintenance of motor evoked potentials despite displacement of the injury, we felt confident in proceeding with the case.

In the setting of such an unstable injury, with severe malalignment related to intraoperative prone positioning, multiple techniques can be used to expeditiously proceed with decompression and stabilization. Rapid decompression at the injured level would allow for some protection 


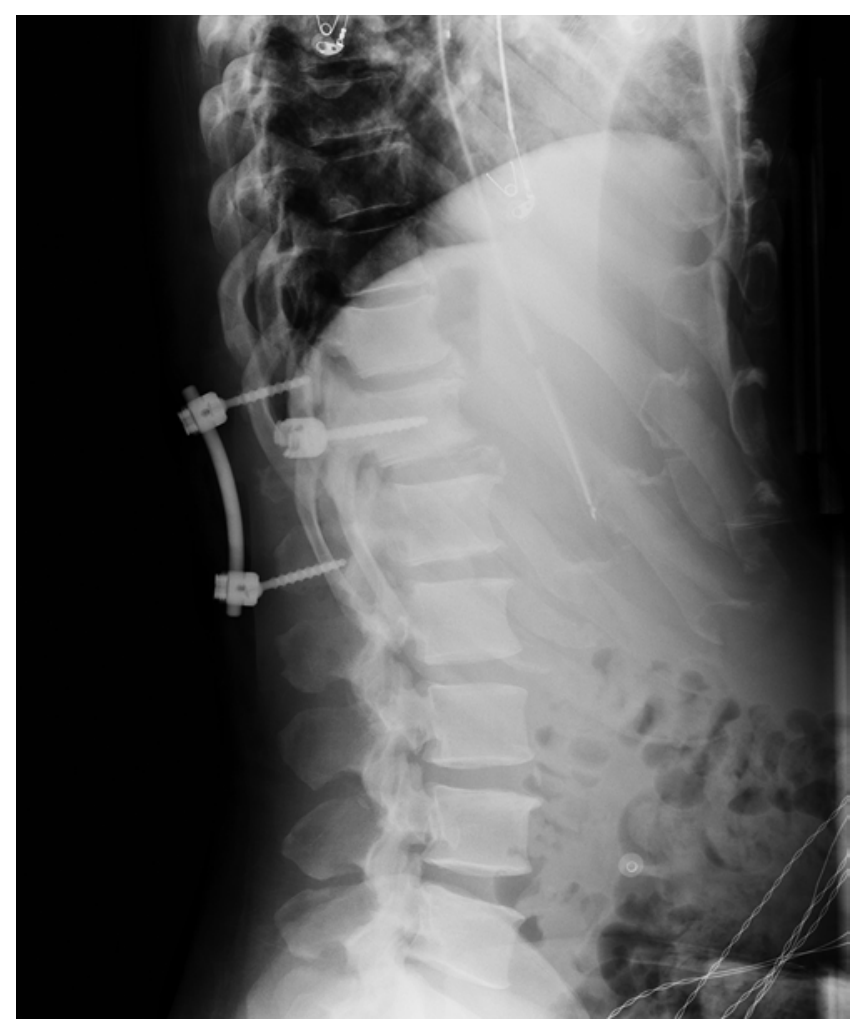

FIG. 3. Lateral intraoperative radiograph showing the temporizing interspinous construct, with screws in the spinous processes of T-11 and L-1. A pedicle screw had been placed in the right pedicle of T-12, cephalad to the injury.

from continued impact on the neural elements, although in this particular case the pattern was so unstable that we were concerned that even gentle manipulation via decompression could cause catastrophic neurological injury. In addition, often an assistant can manually maintain the reduction via clamps while the surgeon expeditiously places definitive fixation; in this case, this technique was initially attempted, but the combination of the patient's dense bone and the instability yielded significant gross motion at the fracture site. Because of this abnormal motion, we decided to place the interspinous process fixation device, as significantly less manipulation was required for this maneuver compared with pedicle cannulation. During placement of the interspinous screws, the assistant maintained the reduction manually in a similar fashion, until the rod was fixed in place.

Though various new methods for spinal fixation have been developed, ${ }^{1,5,8}$ interspinous process screw-and-rod constructs, as detailed in this report, have not previously been described in the literature. Cervical spinous process screws, which are placed perpendicular to the spinous process, have been shown to have pullout strength similar to that of pedicle screws, although these screws are intended for final fixation. ${ }^{4}$ Other novel temporary intraoperative fixation techniques, such as those involving diagonal pedicle screws-and-rod devices during thoracic corpectomy, ${ }^{7}$ have been shown to be effective as a temporizing measure. Primarily, however, the literature

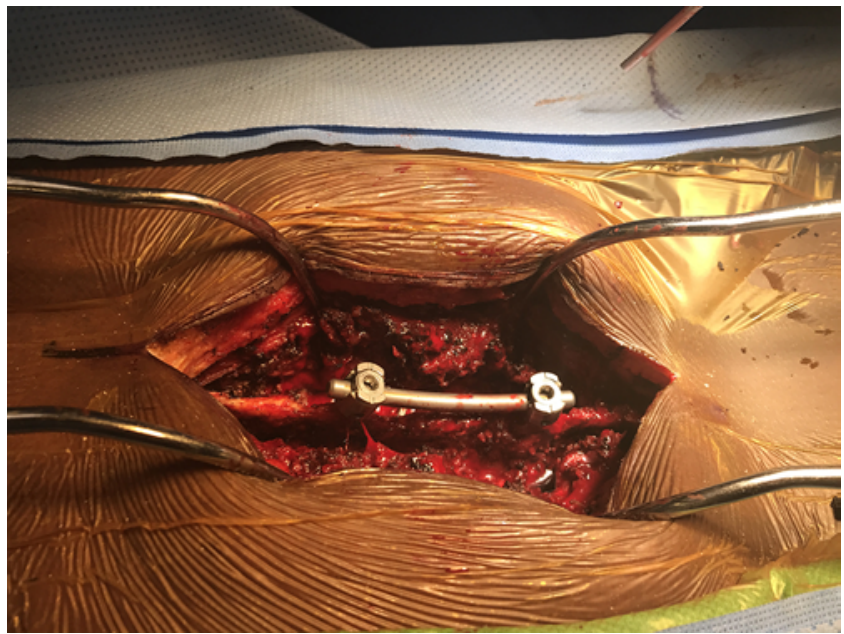

FIG. 4. Intraoperative photograph of the interspinous construct, with the flexion-distraction injury reduced. Figure is available in color online only.

available regarding temporary stabilization of spinal injuries has focused on utilization of minimally invasive techniques without fusion, or "internal bracing," to allow for fracture healing. ${ }^{2}$ We found that placing interspinous process screws with a midline rod provided enough stability to maintain reduction of the severely unstable Chance fracture described in this case and allowed for completion of pedicle screw instrumentation. Furthermore, the location of the midline construct did not preclude instrumentation of the pedicles or access to the lateral aspects of the facets. While a construct of this type has not previously been described or evaluated, we do believe that the simplicity with which temporary fixation was achieved would allow this technique to be applied in other types of unstable injuries (e.g., hyperextension injuries) provided that the spinous processes cephalad and caudal to the injured levels were preserved. When possible, this technique may offer a viable option for temporary spanning fixation in the thoracolumbar spine, and biomechanical investigation may be warranted.
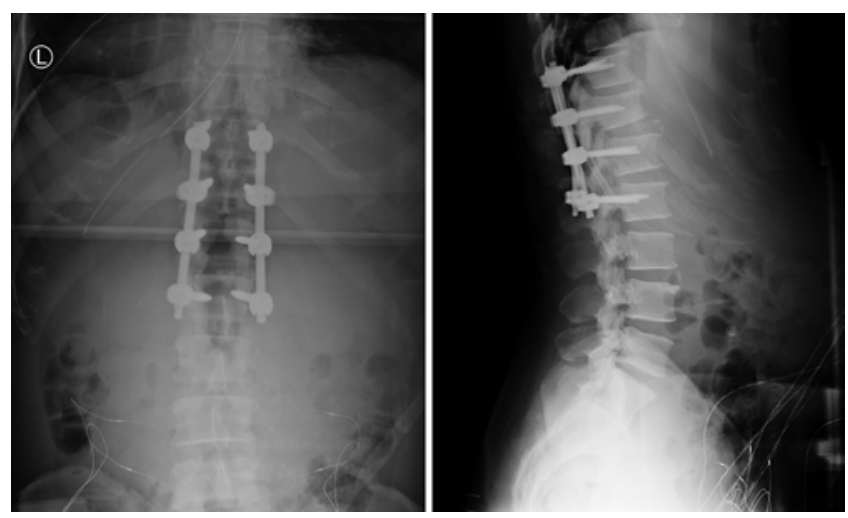

FIG. 5. Postoperative anteroposterior (left) and lateral (right) radiographs of the final construct, consisting of T11-L2 posterior instrumented fusion and laminectomy at T12-L1. 


\section{Acknowledgments}

One author (S.C.W.) is an employee of the United States government and this work was prepared as part of his official duties, and as such there is no copyright to be transferred on his behalf. The views expressed in this article are those of the authors and do not reflect the official policy of the Department of Army/Navy/Air Force, Department of Defense, or US Government.

\section{References}

1. Cho W, Le JT, Shimer AL, Werner BC, Glaser JA, Shen FH: The insertion technique of translaminar screws in the thoracic spine: computed tomography and cadaveric validation. Spine J 15:309-313, 2015

2. Danison AP, Lee DJ, Panchal RR: Temporary stabilization of unstable spine fractures. Curr Rev Musculoskelet Med 10:199-206, 2017

3. Kepler CK, Vaccaro AR, Schroeder GD, Koerner JD, Vialle LR, Aarabi B, et al: The Thoracolumbar AOSpine Injury Score. Global Spine J 6:329-334, 2016

4. Liu GY, Mao L, Xu RM, Ma WH: Biomechanical comparison of pedicle screws versus spinous process screws in $\mathrm{C} 2$ vertebra: A cadaveric study. Indian J Orthop 48:550-554, 2014

5. Menga EN, Jain A, Kebaish KM, Zimmerman SL, Sponseller PD: Anatomic parameters: direct intralaminar screw repair of spondylolysis. Spine (Phila Pa 1976) 39:E153-E158, 2014

6. Radcliff K, Su BW, Kepler CK, Rubin T, Shimer AL, Rihn JA, et al: Correlation of posterior ligamentous complex injury and neurological injury to loss of vertebral body height, kyphosis, and canal compromise. Spine (Phila Pa 1976) 37:1142-1150, 2012

7. Ruban D, O'Toole JE: A novel technique for temporary fixa- tion during posterolateral thoracic corpectomy. J Spinal Disord Tech 24:E66-E70, 2011

8. Sakaura H, Miwa T, Yamashita T, Kuroda Y, Ohwada T: Posterior lumbar interbody fusion with cortical bone trajectory screw fixation versus posterior lumbar interbody fusion using traditional pedicle screw fixation for degenerative lumbar spondylolisthesis: a comparative study. J Neurosurg Spine 25:591-595, 2016

9. Vaccaro AR, Schroeder GD, Kepler CK, Cumhur Oner F, Vialle LR, Kandziora F, et al: The surgical algorithm for the AOSpine thoracolumbar spine injury classification system. Eur Spine J 25:1087-1094, 2016

\section{Disclosures}

The authors report no conflict of interest concerning the materials or methods used in this study or the findings specified in this paper.

\section{Author Contributions}

Conception and design: both authors. Analysis and interpretation of data: Kepler. Drafting the article: both authors. Critically revising the article: both authors. Reviewed submitted version of manuscript: both authors. Approved the final version of the manuscript on behalf of both authors: Wagner.

\section{Correspondence}

Scott C. Wagner, Department of Orthopaedic Surgery, Rothman Institute, Thomas Jefferson University Hospitals, 925 Chestnut St., Philadelphia, PA 19107. email: scott.cameron.wagner@ gmail.com. 